\title{
Some Difference Double Sequence Spaces Defined By Orlicz Function
}

Binod Chandra Tripathy

Mathematical Sciences Division; Institute of Advanced Study in Science and Technology, Paschim Boragaon, Garchuk, Guwahati-781 035, India

e-mail: tripathybc@yahoo.com, tripathybc@rediffmail.com

Bisweshwear Choudhary

Department of Mathematics, University of Botswana, Private Bag No. 0022, Gaborne, Botswana

e-mail : choudhary@mopipi.ub.bw

BIPUL SARMA

Mathematical Sciences Division; Institute of Advanced Study in Science and Technology, Paschim Boragaon, Garchuk, Guwahati-781 035, India

e-mail : sarmabipul01@yahoo.co.in

ABSTRACT. In this article we introduce some difference sequence spaces defined by Orlicz function and study different properties of these spaces like completeness, solidity, symmetricity etc. We establish some inclusion results among them.

\section{Introduction}

Throughout, a double sequence is denoted by $A=<a_{i j}>$. A double sequence is a double infinite array of elements $a_{i j} \in R$ for all $i, j \in N$ and ${ }_{2} w$ will denote the class of all double sequences. The initial works on double sequences is found in Bromwich [2]. Later on it was studied by Hardy [6], Moricz [12], Moricz and Rhoades [13], Tripathy ([16], [17]), Basarir and Sonalcan [1] and many others. Hardy [6] introduced the notion of regular convergence for double sequences. The concept of paranormed sequences was studied by Nakano [14] and Simmons [15] at the initial stage. Later on it was studied by many others. The notion of difference sequence spaces (for single sequences) was introduced by Kizmaz [8] as follows:

$$
Z(\Delta)=\left\{\left(x_{k}\right) \in w:\left(\Delta x_{k}\right) \in Z\right\}
$$

for $Z=c, c_{0}$ and $\ell_{\infty}$, where $\Delta x_{k}=x_{k}-x_{k+1}$, for all $k \in N$.

Received August 14, 2006.

2000 Mathematics Subject Classification: 40A05, 40B05, 46E30.

Key words and phrases: Orlicz function, difference space, completeness, solid space, symmetric space etc. 
The above spaces are Banach spaces, normed by

$$
\left\|\left(x_{k}\right)\right\|=\left|x_{1}\right|+\sup _{k \geq 1}\left|\Delta x_{k}\right|
$$

The notion was further investigated by many workers on sequence spaces.

\section{Definitions and preliminaries}

An Orlicz function $M$ is a mapping $M:[0, \infty) \rightarrow[0, \infty)$ such that it is continuous, non-decreasing and convex with $M(0)=0, M(x)>0$, for $x>0$ and $M(x) \rightarrow \infty$, as $x \rightarrow \infty$.

Lindenstrauss and Tzafriri [10] used the idea of Orlicz function to construct the sequence space

$$
\ell^{M}=\left\{\left(x_{k}\right): \sum_{k=1}^{\infty} M\left(\frac{\left|x_{k}\right|}{\rho}\right)<\infty, \text { for some } \rho>0\right\}
$$

which is a Banach space normed by

$$
\left\|\left(x_{k}\right)\right\|=\inf \left\{\rho>0: \sum_{k=1}^{\infty} M\left(\frac{\left|x_{k}\right|}{\rho}\right) \leq 1\right\} .
$$

The space $\ell^{M}$ is closely related to the space $\ell^{p}$, which is an Orlicz sequence space with $M(x)=|x|^{p}$, for $1 \leq p<\infty$.

An Orlicz function $M$ is said to satisfy the $\Delta_{2}$-condition for all values of $u$, if there exists a constant $K>0$, such that $M(2 u) \leq K(M u), u \geq 0$.

Remark 1. Let $0<\lambda<1$, then $M(\lambda x) \leq \lambda M(x)$, for all $x \geq 0$.

Let $p=\left(p_{k}\right)$ be a positive sequence of real numbers. If $0<p_{k} \leq \sup p_{k}=H$ and $D=\max \left(1,2^{H-1}\right)$, then for $a_{k}, b_{k} \in C$ for all $k \in N$, we have

$$
\left|a_{k}+b_{k}\right|^{p_{k}} \leq D\left\{\left|a_{k}\right|^{p_{k}}+\left|b_{k}\right|^{p_{k}}\right\}
$$

Definition 2.1. A double sequence space $E$ is said to be solid if $<\alpha_{i j} a_{i j}>\in E$ whenever $\left\langle a_{i j}>\in E\right.$, for all double sequences $\left\langle\alpha_{i j}>\right.$ of scalars with $\left|\alpha_{i j}\right| \leq 1$, for all $i, j \in N$.

Definition 2.2. Let $K=\left\{\left(n_{i}, k_{j}\right): i, j \in N ; n_{1}<n_{2}<n_{3}<\ldots\right.$ and $k_{1}<k_{2}<$ $\left.k_{3}<\ldots\right\} \subseteq N \times N$ and $E$ be a double sequence space. A $K$-step space of $E$ is a sequence space

$$
\lambda_{K}^{E}=\left\{<a_{n_{i} k_{j}}>\epsilon_{2} w:<a_{n k}>\in E\right\} .
$$


A canonical pre-image of a sequence $<a_{n_{i} k_{j}}>\in E$ is a sequence $<b_{n k}>\in E$ defined as follows:

$$
b_{n k}= \begin{cases}a_{n k}, & \text { if }(n, k) \in K, \\ 0, & \text { otherwise }\end{cases}
$$

A canonical pre-image of a step space $\lambda_{K}^{E}$ is a set of canonical pre-images of all elements in $\lambda_{K}^{E}$.

Definition 2.3. A double sequence space $E$ is said to be monotone if it contains the canonical pre-images of all its step spaces.

Remark 2. From the above notions, it follows that "If a sequence space $E$ is solid then $E$ is monotone".

Definition 2.4. A double sequence space $E$ is said to be symmetric if $\left\langle a_{i j}\right\rangle \in E$ implies $<a_{\pi(i) \pi(j)}>\in E$, where $\pi$ is a permutation of $N$.

Let $M$ be an Orlicz function and $p=<p_{i j}>$ be a double sequence of strictly positive real numbers. We introduce the following sequence spaces.

$$
\begin{aligned}
& { }_{2} W(M, \Delta, p)=\left\{<a_{i j}>\in{ }_{2} w: \lim _{m, n} \frac{1}{m n} \sum_{i=1}^{m} \sum_{j=1}^{n}\left(M\left(\frac{\left|\Delta a_{i j}-L\right|}{\rho}\right)\right)^{p_{i j}}=0,\right. \\
& \text { for some } \rho>0 \text { and } L\} . \\
& { }_{2} W_{0}(M, \Delta, p)=\left\{<a_{i j}>\in{ }_{2} w: \lim _{m, n} \frac{1}{m n} \sum_{i=1}^{m} \sum_{j=1}^{n}\left(M\left(\frac{\left|\Delta a_{i j}\right|}{\rho}\right)\right)^{p_{i j}}=0,\right. \\
& \text { for some } \rho>0\} . \\
& { }_{2} W_{\infty}(M, \Delta, p)=\left\{<a_{i j}>\in{ }_{2} w: \sup _{m, n} \frac{1}{m n} \sum_{i=1}^{m} \sum_{j=1}^{n}\left(M\left(\frac{\left|\Delta a_{i j}\right|}{\rho}\right)\right)^{p_{i j}}<\infty,\right. \\
& \text { for some } \rho>0\} .
\end{aligned}
$$

\section{Main results}

Theorem 3.1. Let $p=<p_{i j}>$ be bounded. The classes of sequences ${ }_{2} W(M, \Delta, p)$, ${ }_{2} W_{0}(M, \Delta, p)$ and ${ }_{2} W_{\infty}(M, \Delta, p)$ are linear spaces.

Theorem 3.2. The sequence spaces ${ }_{2} W(M, \Delta, p),{ }_{2} W_{0}(M, \Delta, p)$ and $_{2} W_{\infty}(M, \Delta, p)$ are paranormed sequence spaces, paranormed by

$$
g\left(<a_{i j}>\right)=\sup _{i}\left|a_{i 1}\right|+\sup _{j}\left|a_{1 j}\right|+\inf \left\{\rho^{\frac{p_{i j}}{J}}>0: \sup _{i, j}\left(M\left(\frac{\left|\Delta a_{i j}\right|}{\rho}\right)\right) \leq 1\right\},
$$


where $J=\max (1, H), H=\sup _{i, j} p_{i j}$.

Proof. Clearly $g(0)=0, \quad g\left(-<a_{i j}>\right)=g\left(<a_{i j}>\right)$. Let $<a_{i j}>,<b_{i j}>\epsilon$ ${ }_{2} W_{\infty}(M, \Delta, p)$.

Then there exist some $\rho_{1}, \rho_{2}>0$ such that

$$
\sup _{i, j}\left(M\left(\frac{\left|\Delta a_{i j}\right|}{\rho_{1}}\right)\right) \leq 1 \text { and } \sup _{i, j}\left(M\left(\frac{\left|\Delta b_{i j}\right|}{\rho_{2}}\right)\right) \leq 1 .
$$

Let $\rho=\rho_{1}+\rho_{2}$. Then we have,

$$
\begin{aligned}
& \sup _{i, j}\left(M\left(\frac{\left|\Delta a_{i j}+\Delta b_{i j}\right|}{\rho}\right)\right) \\
\leq & \frac{\rho_{1}}{\rho_{1}+\rho_{2}} \sup _{i, j}\left(M\left(\frac{\left|\Delta a_{i j}\right|}{\rho_{1}}\right)\right)+\frac{\rho_{2}}{\rho_{1}+\rho_{2}} \sup _{i, j}\left(M\left(\frac{\left|\Delta b_{i j}\right|}{\rho_{2}}\right)\right) \leq 1 .
\end{aligned}
$$

Now

$$
\begin{aligned}
& g\left(<a_{i j}>+<b_{i j}>\right)=\left|a_{i 1}+b_{i 1}\right|+\left|a_{1 j}+b_{1 j}\right| \\
& +\inf \left\{\left(\rho_{1}+\rho_{2}\right)^{\frac{p_{i j}}{J}}>0: \sup _{i, j}\left(M\left(\frac{\mid \Delta a_{i j}+\Delta b_{i j}}{\rho_{1}+\rho_{2}}\right)\right) \leq 1\right\} \\
\leq & \left|a_{i 1}\right|+\left|b_{i 1}\right|+\inf \left\{\rho_{1}^{\frac{p_{i j}}{j}}>0: \sup _{i, j}\left(M\left(\frac{\left|\Delta a_{i j}\right|}{\rho_{1}}\right)\right) \leq 1\right\} \\
& +\left|a_{1 j}\right|+\left|b_{1 j}\right|+\inf \left\{\rho_{2}^{\frac{p_{i j}}{J}}>0: \sup _{i, j}\left(M\left(\frac{\left|\Delta b_{i j}\right|}{\rho_{2}}\right)\right) \leq 1\right\} \\
= & g\left(<a_{i j}>\right)+g\left(<b_{i j}>\right) .
\end{aligned}
$$

Let $\eta \in C$, then the continuity of the product follows from the following equality.

$$
\begin{aligned}
& g\left(\eta<a_{i j}>\right) \\
= & \left|\eta a_{i 1}\right|+\left|\eta a_{i 1}\right|+\inf \left\{\rho^{\frac{p_{i j}}{J}}: \sup _{i, j}\left(M\left(\frac{\left|\Delta \eta a_{i j}\right|}{\rho}\right)\right) \leq 1, \rho>0\right\} \\
= & |\eta|\left|a_{i 1}\right|+|\eta|\left|a_{i 1}\right|+\inf \left\{(|\eta| r)^{\frac{p_{i j}}{J}}: \sup _{i, j}\left(M\left(\frac{\left|\Delta \eta a_{i j}\right|}{r}\right)\right) \leq 1, r>0\right\},
\end{aligned}
$$

where $\frac{1}{r}=\frac{|\eta|}{\rho}$.

Theorem 3.3. The sequence spaces ${ }_{2} W(M, \Delta, p),{ }_{2} W_{0}(M, \Delta, p)$ and $_{2} W_{\infty}(M, \Delta, p)$ are complete paranormed spaces, paranormed by $g$.

Proof. Let $\left\langle a_{i j}^{s}\right\rangle$ be a Cauchy sequence in ${ }_{2} W_{\infty}(M, \Delta, p)$. Then $g\left(\left\langle a_{i j}^{s}-a_{i j}^{t}\right\rangle\right.$ )$\rightarrow 0$ as $s, t \rightarrow \infty$.

For a given $\epsilon>0$, choose $r>0$ and $x_{0}>0$ be such that $\frac{\epsilon}{r x_{0}}>0$ and $M\left(\frac{r x_{0}}{2}\right) \geq 1$. 
Now $\left.g\left(<a_{i j}^{s}-a_{i j}^{t}\right\rangle\right) \rightarrow 0$ as $s, t \rightarrow \infty$ implies that there exists $m_{0} \in N$ such that

$$
g\left(<a_{i j}^{s}-a_{i j}^{t}>\right)<\frac{\epsilon}{r x_{0}} \quad \text { for all } s, t \geq m_{0} .
$$

$\Rightarrow \sup _{i}\left|a_{i 1}^{s}-a_{i 1}^{t}\right|+\sup _{j}\left|a_{1 j}^{s}-a_{1 j}^{t}\right|+$

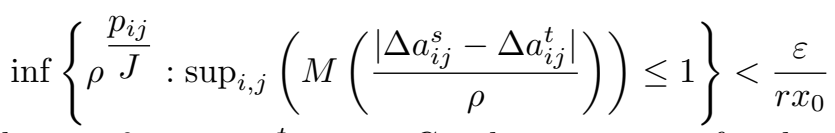

This shows that $\left\langle a_{i 1}^{s}\right\rangle,\left\langle a_{1 j}^{t}\right\rangle$ are Cauchy sequences of real numbers. As the set of real numbers is complete so there exists real numbers $a_{i 1}, a_{1 j}$ such that

$$
\lim _{s \rightarrow \infty} a_{i 1}^{s}=a_{i 1}, \quad \lim _{s \rightarrow \infty} a_{1 j}^{s}=a_{1 j} .
$$

Now from (1) we have,

$$
\begin{aligned}
& \left(M\left(\frac{\left|\Delta a_{i j}^{s}-\Delta a_{i j}^{t}\right|}{\rho}\right)\right) \leq 1 \\
\Rightarrow & \sup _{i, j}\left(M\left(\frac{\left|\Delta a_{i j}^{s}-\Delta a_{i j}^{t}\right|}{\rho}\right)\right) \leq 1 \leq M\left(\frac{r x_{0}}{2}\right) \\
\Rightarrow & \frac{\left|\Delta a_{i j}^{s}-\Delta a_{i j}^{t}\right|}{g\left(<a_{i j}^{s}-a_{i j}^{t}>\right)} \leq \frac{r x_{0}}{2} \\
\Rightarrow & \left|\Delta a_{i j}^{s}-\Delta a_{i j}^{t}\right|<\frac{r x_{0}}{2} \cdot \frac{\epsilon}{r x_{0}}=\frac{\epsilon}{2} .
\end{aligned}
$$

This implies $<\Delta a_{i j}^{s}>$ is a Cauchy sequence of real numbers. Let $\lim _{s \rightarrow \infty} \Delta a_{i j}^{s}=y_{i j}$ for all $i, j \in N$. Now $\Delta a_{11}^{s}=a_{11}^{s}-a_{12}^{s}-a_{21}^{s}+a_{22}^{s}$ and so

$$
\lim _{s \rightarrow \infty} a_{22}^{s}=\lim _{s \rightarrow \infty}\left(\Delta a_{11}^{s}-a_{11}^{s}+a_{12}^{s}+a_{21}^{s}\right)=y_{11}-a_{11}+a_{12}+a_{21} .
$$

Hence $\lim _{s \rightarrow \infty} a_{22}^{s}$ exists. Proceeding in this way we conclude that $\lim _{s \rightarrow \infty} a_{i j}^{s}$ exists and thus $\lim _{s \rightarrow \infty} \Delta a_{i j}^{s}$ exists. Using continuity of $M$, we have

$$
\begin{aligned}
& \limsup _{t \rightarrow \infty}\left(M\left(\frac{\left|\Delta a_{i j}^{s}-\Delta a_{i j}\right|}{\rho}\right)\right) \leq 1 \\
\Rightarrow & \lim _{t \rightarrow \infty} \sup _{i, j}\left(M\left(\frac{\left|\Delta a_{i j}^{s}-\Delta a_{i j}\right|}{\rho}\right)\right) \leq 1 .
\end{aligned}
$$

Let $s \geq m_{0}$, then taking infimum of such $\rho$ 's we have $g\left(<a_{i j}^{s}-a_{i j}>\right)<\epsilon$.

Thus $<a_{i j}^{s}-a_{i j}>\in{ }_{2} W_{\infty}(M, \Delta, p)$. By linearity of the space ${ }_{2} W_{\infty}(M, \Delta, p)$ we have $\left\langle a_{i j}\right\rangle \in{ }_{2} W_{\infty}(M, \Delta, p)$. Hence ${ }_{2} W_{\infty}(M, \Delta, p)$ is complete. 


\section{Proposition 3.4.}

(i) ${ }_{2} W(M, \Delta, p) \subset{ }_{2} W_{\infty}(M, \Delta, p)$,

(ii) ${ }_{2} W_{0}(M, \Delta, p) \subset{ }_{2} W_{\infty}(M, \Delta, p)$.

The inclusions are strict.

Proof. The proof is easy, so omitted.

Theorem 3.5. The spaces ${ }_{2} W(M, \Delta, p)$ and ${ }_{2} W_{0}(M, \Delta, p)$ are nowhere dense subset of ${ }_{2} W_{\infty}(M, \Delta, p)$.

Proof. The proof is obvious in view of Theorem 3.3 and Theorem 3.4.

Theorem 3.6. If $\sup \frac{p_{i j}}{p_{u v}}<\infty$, for all $i \geq u, j \geq v$, then ${ }_{2} W(M, p) \subset{ }_{2} W_{0}(M, \Delta, p)$. The inclusion is strict.

Proof. Let $\left.<a_{i j}\right\rangle \in{ }_{2} W(M, p)$. Then

$$
\lim _{m, n} \frac{1}{m n} \sum_{i=1}^{m} \sum_{j=1}^{n}\left(M\left(\frac{\left|a_{i j}-L\right|}{\rho}\right)\right)^{p_{i j}}=0, \text { for some } \rho>0 \text { and } L
$$

Since $\sup \frac{p_{i j}}{p_{u v}}<\infty$, so there exists $K>0$ such that $p_{i j}<K$. $p_{u v}$ for all $i \geq u, j \geq v$.

Thus from (2) we have,

$$
\begin{aligned}
\lim _{m, n} \frac{1}{m n} \sum_{i=1}^{m} \sum_{j=1}^{n}\left(M\left(\frac{\left|a_{i j}-L\right|}{\rho}\right)\right)^{p_{i, j+1}} & =0, \\
\lim _{m, n} \frac{1}{m n} \sum_{i=1}^{m} \sum_{j=1}^{n}\left(M\left(\frac{\left|a_{i j}-L\right|}{\rho}\right)\right)^{p_{i+1, j}} & =0, \\
\text { and } \lim _{m, n} \frac{1}{m n} \sum_{i=1}^{m} \sum_{j=1}^{n}\left(M\left(\frac{\left|a_{i j}-L\right|}{\rho}\right)\right)^{p_{i+1, j+1}} & =0,
\end{aligned}
$$

Now for $\left|\Delta a_{i j}\right|=\left|a_{i j}-a_{i, j+1}-a_{i+1, j}+a_{i+1, j+1}+L-L+L-L\right|$ we have,

$$
\begin{aligned}
& \lim _{m, n} \frac{1}{m n} \sum_{i=1}^{m} \sum_{j=1}^{n}\left(M\left(\frac{\left|\Delta a_{i j}\right|}{\rho}\right)\right)^{p_{i, j}} \\
\leq & \lim _{m, n} \frac{1}{m n} \sum_{i=1}^{m} \sum_{j=1}^{n}\left(M \left(\frac{\left|a_{i j}-L\right|}{\rho}+\frac{\left|a_{i+1, j}-L\right|}{\rho}+\frac{\left|a_{i, j+1}-L\right|}{\rho}\right.\right. \\
& \left.\left.+\frac{\left|a_{i+1, j+1}-L\right|}{\rho}\right)\right)^{p_{i j}}
\end{aligned}
$$




$$
\begin{aligned}
\leq \quad & D^{2} \cdot \lim _{m, n} \frac{1}{m n} \sum_{i=1}^{m} \sum_{j=1}^{n}\left\{\left(M\left(\frac{\left|a_{i j}-L\right|}{\rho}\right)\right)^{p_{i j}}+\left(M\left(\frac{\left|a_{i+1, j}-L\right|}{\rho}\right)\right)^{p_{i j}}\right. \\
& \left.+\left(M\left(\frac{\left|a_{i, j+1}-L\right|}{\rho}\right)\right)^{p_{i j}}+\left(M\left(\frac{\left|a_{i+1, j+1}-L\right|}{\rho}\right)\right)^{p_{i j}}\right\} \\
\leq \quad & D^{2} \cdot \lim _{m, n} \frac{1}{m n} \sum_{i=1}^{m} \sum_{j=1}^{n}\left\{\left(M\left(\frac{\left|a_{i j}-L\right|}{\rho}\right)\right)^{p_{i j}}+\left(M\left(\frac{\left|a_{i+1, j}-L\right|}{\rho}\right)\right)^{p_{i+1, j}}\right. \\
& \left.+\left(M\left(\frac{\left|a_{i, j+1}-L\right|}{\rho}\right)\right)^{p_{i, j+1}}+\left(M\left(\frac{\left|a_{i+1, j+1}-L\right|}{\rho}\right)\right)^{p_{i+1, j+1}}\right\}=0 .
\end{aligned}
$$

Thus $\left\langle a_{i j}>\in{ }_{2} W_{0}(M, \Delta, p)\right.$ and hence ${ }_{2} W(M, p) \subset_{2} W_{0}(M, \Delta, p)$.

The inclusion is strict follows from the following example.

Example 3.1. Let $M(x)=x^{p}, p_{i j}=1$ for all $i$ odd and for all $j \in N$ and $p_{i j}=2$ otherwise.

Consider the sequence $\left\langle a_{i j}>\right.$ defined by $a_{i j}=i+j$ for all $i, j \in N$. We have $\Delta a_{i j}=0$ for all $i, j \in N$. Hence $<a_{i j}>\in{ }_{2} W(M, \Delta, p)$ but $<a_{i j}>\notin{ }_{2} W(M, p)$.

\section{Theorem 3.7.}

(i) If $0<\inf p_{i j} \leq p_{i j}<1$, then ${ }_{2} W(M, \Delta, p) \subseteq{ }_{2} W(M, \Delta)$.

(ii) If $1<p_{i j} \leq \sup p_{i j}<\infty$, then ${ }_{2} W(M, \Delta) \subseteq{ }_{2} W(M, \Delta, p)$.

Proof. The first part of the result follows from the inequality

$$
\frac{1}{m n} \sum_{i=1}^{m} \sum_{j=1}^{n}\left(M\left(\frac{\left|\Delta a_{i j}-L\right|}{\rho}\right)\right) \leq \frac{1}{m n} \sum_{i=1}^{m} \sum_{j=1}^{n}\left(M\left(\frac{\left|\Delta a_{i j}-L\right|}{\rho}\right)\right)^{p_{i, j}},
$$

and the second part of the result follows from the inequality

$$
\frac{1}{m n} \sum_{i=1}^{m} \sum_{j=1}^{n}\left(M\left(\frac{\left|\Delta a_{i j}-L\right|}{\rho}\right)\right)^{p_{i, j}} \leq \frac{1}{m n} \sum_{i=1}^{m} \sum_{j=1}^{n}\left(M\left(\frac{\left|\Delta a_{i j}-L\right|}{\rho}\right)\right) .
$$

Theorem 3.8. Let $M_{1}$ and $M_{2}$ be two Orlicz functions. Then

$$
{ }_{2} W\left(M_{1}, \Delta, p\right) \cap{ }_{2} W\left(M_{2}, \Delta, q\right) \subseteq{ }_{2} W\left(M_{1}+M_{2}, \Delta, q\right) .
$$


Proof. Let $<a_{i j}>\in{ }_{2} W\left(M_{1}, \Delta, p\right) \cap{ }_{2} W\left(M_{2}, \Delta, q\right)$. Then

$$
\begin{aligned}
& \lim _{m, n} \frac{1}{m n} \sum_{i=1}^{m} \sum_{j=1}^{n}\left(M_{1}\left(\frac{\left|\Delta a_{i j}-L\right|}{\rho_{1}}\right)\right)^{p_{i, j}}=0, \text { for some } \rho_{1}>0 \\
& \lim _{m, n} \frac{1}{m n} \sum_{i=1}^{m} \sum_{j=1}^{n}\left(M_{2}\left(\frac{\left|\Delta a_{i j}-L\right|}{\rho_{2}}\right)\right)^{p_{i, j}}=0, \text { for some } \rho_{2}>0 .
\end{aligned}
$$

Let $\rho=\max \left\{\rho_{1}, \rho_{2}\right\}$. The result follows from the following inequality.

$$
\begin{aligned}
& \sum_{i=1}^{m} \sum_{j=1}^{n}\left(\left(M_{1}+M_{2}\right)\left(\frac{\left|\Delta a_{i j}-L\right|}{\rho}\right)\right)^{p_{i, j}} \\
\leq & D\left\{\sum_{i=1}^{m} \sum_{j=1}^{n}\left(M_{1}\left(\frac{\left|\Delta a_{i j}-L\right|}{\rho_{1}}\right)\right)^{p_{i, j}}+\sum_{i=1}^{m} \sum_{j=1}^{n}\left(M_{2}\left(\frac{\left|\Delta a_{i j}-L\right|}{\rho_{2}}\right)\right)^{p_{i, j}}\right\} .
\end{aligned}
$$

Theorem 3.9. The sequence space ${ }_{2} W_{\infty}(M, \Delta, p)$ is solid and hence monotone. Proof. Let $<a_{i j}>\in{ }_{2} W_{\infty}(M, \Delta, p)$ and $\left\langle\alpha_{i j}>\right.$ be a scalar sequence such that $\left|\alpha_{i j}\right| \leq 1$ for all $i, j \in N$. Now

$$
\begin{aligned}
& M\left(\frac{\left|\alpha_{i j} \Delta a_{i j}\right|}{\rho}\right) \leq M\left(\frac{\left|\Delta a_{i j}\right|}{\rho}\right) \\
\Rightarrow & \left(M\left(\frac{\left|\alpha_{i j} \Delta a_{i j}\right|}{\rho}\right)\right)^{p_{i j}} \leq\left(M\left(\frac{\left|\Delta a_{i j}\right|}{\rho}\right)\right)^{p_{i j}} \\
\Rightarrow & \sup _{m, n} \frac{1}{m n} \sum_{i=1}^{m} \sum_{j=1}^{n}\left(M\left(\frac{\left|\alpha_{i j} \Delta a_{i j}\right|}{\rho}\right)\right)^{p_{i j}} \\
\leq & \sup _{m, n} \frac{1}{m n} \sum_{i=1}^{m} \sum_{j=1}^{n}\left(M\left(\frac{\left|\Delta a_{i j}\right|}{\rho}\right)\right)^{p_{i j}}<\infty .
\end{aligned}
$$

Result 3.10. The sequence spaces ${ }_{2} W(M, \Delta, p)$ and ${ }_{2} W_{0}(M, \Delta, p)$ are not monotone and hence are not solid.

Proof. The result follows from the following example.

Example 3.2. Let $M(x)=x^{p}, p \geq 1$. Then the double sequence $\left\langle a_{i j}\right\rangle$ defined by $a_{i j}=1$, for all $i, j \in N$ belongs to ${ }_{2} W(M, \Delta, p)$ and ${ }_{2} W_{0}(M, \Delta, p)$. Consider its pre-image $<b_{i j}>$ defined as

$$
b_{i j}= \begin{cases}a_{i j}, & \text { if } i+j \text { is odd } \\ 0, & \text { otherwise }\end{cases}
$$


Then $<b_{i j}>$ belongs neither to ${ }_{2} W(M, \Delta, p)$ nor to ${ }_{2} W_{0}(M, \Delta, p)$. Hence the spaces ${ }_{2} W(M, \Delta, p)$ and ${ }_{2} W_{0}(M, \Delta, p)$ are not monotone and by Remark 3 these are not solid also.

Result 3.11. The sequence spaces ${ }_{2} W(M, \Delta, p),{ }_{2} W_{0}(M, \Delta, p)$ and ${ }_{2} W_{\infty}(M, \Delta, p)$ are are not symmetric.

Proof. To prove the results consider the following examples.

Example 3.3. Let $M(x)=x^{2}$. Consider the sequence $<a_{i j}>$ defined by

$$
a_{i j}= \begin{cases}1, & \text { if } i \text { is odd for all } j \in N, \\ -1, & \text { otherwise }\end{cases}
$$

Then $\Delta a_{i j}=0$ for all $i, j \in N$. Let $\left\langle b_{i j}>\right.$ be a rearrangement of the sequence $<a_{i j}>$ defined by

$$
b_{i j}= \begin{cases}-1, & \text { if } i+j \text { is even } \\ 1, & \text { otherwise. }\end{cases}
$$

Then

$$
\Delta b_{i j}= \begin{cases}-4, & \text { if } i+j \text { is even } \\ 4, & \text { otherwise. }\end{cases}
$$

Here $<a_{i j}>\in{ }_{2} W_{0}(M, \Delta, p) \subseteq{ }_{2} W(M, \Delta, p)$, but $<b_{i j}>\notin{ }_{2} W(M, \Delta, p)$.

Example 3.4. Let $M(x)=x^{p}, p \geq 1, \quad p_{i j}=2$ for all $i, j \in N$. Consider the sequence $<a_{i j}>$ defined by

$$
a_{i j}= \begin{cases}0, & \text { if } i \text { is even, for all } j \in N, \\ i, & \text { otherwise. }\end{cases}
$$

Then $\Delta a_{i j}=0$ for all $i, j \in N$.

Let $\left\langle b_{i j}\right\rangle$ be a rearrangement of the sequence $<a_{i j}>$ defined by

$$
b_{i j}= \begin{cases}0, & \text { if } i+j \text { is odd } \\ i, & \text { otherwise }\end{cases}
$$

Then $\Delta b_{i j}= \begin{cases}2 i+1, & \text { if } i+j \text { is even, } \\ -2 i-1, & \text { otherwise. }\end{cases}$

Here $<a_{i j}>\in{ }_{2} W_{\infty}(M, \Delta, p)$, but $<b_{i j}>\notin{ }_{2} W_{\infty}(M, \Delta, p)$. 


\section{References}

[1] M. Basarir, and O. Sonalcan, On some double sequence spaces, J. Indian Acad. Math., 21(2)(1999), 193-200.

[2] Bromwich T.J.I ${ }_{A}$ : : An Introduction to the Theory of Infinite Series, MacMillan and Co.Ltd., New york 1965.

[3] A. Esi, Some new sequence spaces defined by Orlicz functions, Bull. Inst. Math. Acad. Sinica., 27(1)(1999),71-76.

[4] A. Esi and M. Et, Some new sequence spaces defined by a sequence of Orlicz functions, Indian J. Pure. Appl. Math., 31(8)(2000), 967-972.

[5] M. Et, On some new Orlicz sequence spaces, J. Analysis, 9(2001), 21-28.

[6] G. H. Hardy, On the convergence of certain multiple series, Proc. Camb. Phil. Soc., 19(1917), 86-95.

[7] P. K. Kamthan and M. Gupta, Sequence Spaces and Series, Marcel Dekker, 1980.

[8] H. Kizmaz, On certain sequence spaces, Canad. Math. Bull., 24(1981), 169 -176.

[9] M. A. Krasnoselkii and Y. B. Rutitsky, Convex function and Orlicz Spaces, Groningen, Netherlands, 1961.

[10] J. Lindenstrauss and L. Tzafriri, On Orlicz sequence spaces, Israel J. Math., 10(1971), 379-390.

[11] I. J. Maddox, Spaces of strongly summable sequences, Quart. Jour. Math.(Oxford 2nd Ser), 18(72)(1976), 345-355.

[12] F. Moricz, Extension of the spaces $c$ and $c_{0}$ from single to double sequences, Acta. Math., Hungerica., 57(1-2)(1991), 129-136.

[13] F. Moricz and B. E. Rhoades, Almost convergence of double sequences and strong regularity of summability matrices, Math. Proc. Camb. Phil. Soc., 104(1988), 283294.

[14] H. Nakano, Modular sequence spaces, Proc. Japan Acad., 27(1951), 508-512.

[15] S. Simons, The sequence spaces $\ell\left(p_{\nu}\right)$ and $m\left(p_{\nu}\right)$, Proc. London Math. Soc., 15(3)(1965), 422-436.

[16] B. C. Tripathy, Generalized difference paranormed statistically convergent sequences defined by Orlicz function in a locally convex spaces, Soochow J. Math., 30(4)(2004), 431-446.

[17] B. C. Tripathy, Statistically convergent double sequences, Tamkang Jour. Math., 34(3)(2003), 231-237.

[18] B. C. Tripathy and B. Sarma, Statistically convergent double sequence spaces defined by Orlicz functions, Soochow J. Math., 32(2)(2006), 211-221.

[19] A. Zygumd, Trigonometric Series, vol II, Cambridge, 1993. 